\title{
The Construction of the Fiber-SiPM beam monitor system of the R484 and R582 Experiments at the RIKEN-RAL Muon facility.
}

\author{
M.Bonesini, ${ }^{a, 1}$ R.Bertoni, ${ }^{a}$ F.Chignoli, ${ }^{a}$ R.Mazza, ${ }^{a}$ T.Cervi, $^{b}$ A.deBari, ${ }^{b}$ A.Menegolli, ${ }^{b}$ \\ M.C.Prata, ${ }^{b}$ M.Rossella, ${ }^{b}$ L.Tortora, ${ }^{c}$ R.Carbone, ${ }^{d}$ E.Mocchiutti, ${ }^{d}$ A.Vacchi, ${ }^{d, 2}$ E.Vallazza, ${ }^{d}$ \\ G.Zampa ${ }^{d}$ \\ ${ }^{a}$ Sezione INFN Milano Bicocca, Dipartimento di Fisica G. Occhialini, Universitá di Milano Bicocca, \\ Piazza Scienza 3, Milano, Italy \\ ${ }^{b}$ Sezione INFN Pavia, Dipartimento di Fisica, Universitá di Pavia, \\ via A. Bassi 6, Pavia, Italy \\ c Sezione INFN Roma Tre, \\ via della Vasca Navale 84, Roma, Italy \\ ${ }^{d}$ Sezione INFN Trieste, \\ via Padriciano 99, Trieste, Italy \\ E-mail: maurizio.bonesini@mib.infn.it
}

\begin{abstract}
The scintillating fiber-SiPM beam monitor detectors, designed to deliver beam informations for the R484 and R582 experiments at the high intensity, low energy pulsed muon beam at the RIKEN-RAL facility, have been successfully constructed and operated. Details on their construction and first performances in beam are reported.
\end{abstract}

KEYwORDs: Beam-line instrumentation; particle tracking detectors; scintillators.

${ }^{1}$ Corresponding author.

${ }^{2}$ Also at Department of Mathematics, Computer Science and Physics, University of Udine, Udine, Italy. 


\section{Contents}

1 Introduction 1

2 Construction of the R484 and R582 beam hodoscopes 2

2.1 The R484 experiment beam hodoscope 2

2.2 The R582 experiment beam hodoscope 5

3 Preliminary performances at RIKEN-RAL. $\quad 8$

4 Conclusions $\quad 10$

\section{Introduction}

The FAMU experiment at RAL, see reference [1] for further details, aims at the measurement of the hyperfine splitting (HFS) in the ground state (1S) of the muonic hydrogen [2], thus providing a high accuracy determination of the proton Zemach radius [3]. The experiment may thus contribute to solve the so-called "proton radius puzzle": a $7 \sigma$ disagreement between the proton charge radius as determined from electrons or muons [4]. For this experiment, an important issue is the optimal
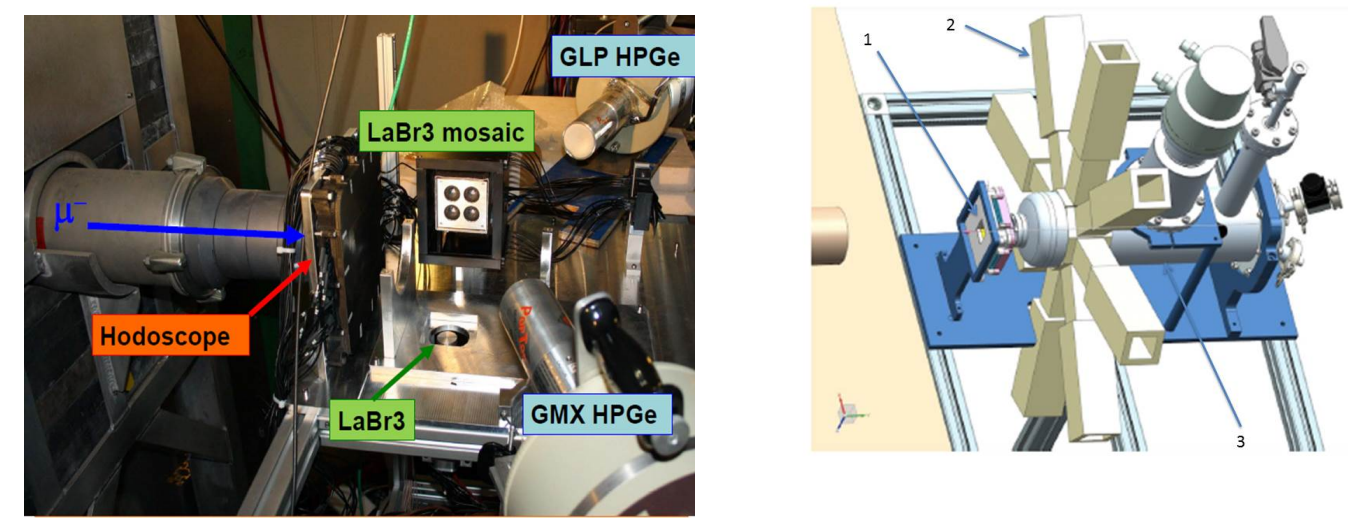

Figure 1. Left panel: picture of the setup for the 2014 data-taking (R484). Right panel: layout of the setup for the 2015-2016 data-taking (R582); 1) is the beam hodoscope, 2) the crown of $8 \mathrm{LaBr}_{3}$ crystals read by photomultipliers and 3) the cryogenic target. The four HPGe detectors, also used in this run, are not shown.

steering of the impinging high intensity pulsed muon beam onto the hydrogen target, to maximize the muonic hydrogen production rate. A system of two beam hodoscopes has been developed for this scope. The first one is based on square $3 \times 3 \mathrm{~mm}^{2}$ scintillating fibers read by SiPM, for the R484 experiment, which had a data taking in June 2014, while the second one is based on square $1 \times 1 \mathrm{~mm}^{2}$ scintillating fibers read by SiPM, for the R582 experiment, which instead had a data 
taking in December 2015 and February 2016. A schematic layout of both experimental setup is shown in figure 1.

The RIKEN-RAL muon facility at Rutherford Appleton Laboratory (UK) provides high intensity pulsed muon beams at four experimental ports, as shown in figure 2 . The ISIS primary proton beam at $800 \mathrm{MeV} / \mathrm{c}$, with a $50 \mathrm{~Hz}$ repetition rate, impinges from the left on a secondary carbon target producing pions and then high intensity low energy pulsed muon beams. Surface $\mu^{+}$(20-30 $\mathrm{MeV} / \mathrm{c})$ are produced by pions stopping close to the target surface, while decay $\mu^{+} / \mu^{-}(20-120$ $\mathrm{MeV} / \mathrm{c}$ ) are produced from pion decay outside the target.

The muon beams reflect the primary beam structure: two pulses with a 70 ns FWHM and a $320 \mathrm{~ns}$ peak to peak distance are delivered. The FAMU experiment makes use of a negative decay muon beam at $\sim 60 \mathrm{MeV} / \mathrm{c}$. The intensity is around $8 \times 10^{4} \mu^{-} / \mathrm{s}$ in a typical size $4 \times 4 \mathrm{~cm}^{2}$ as shown in the right panel of figure 2 . The energy spread is around $10 \%$ and the angular divergence around $60 \mathrm{mrad}$.
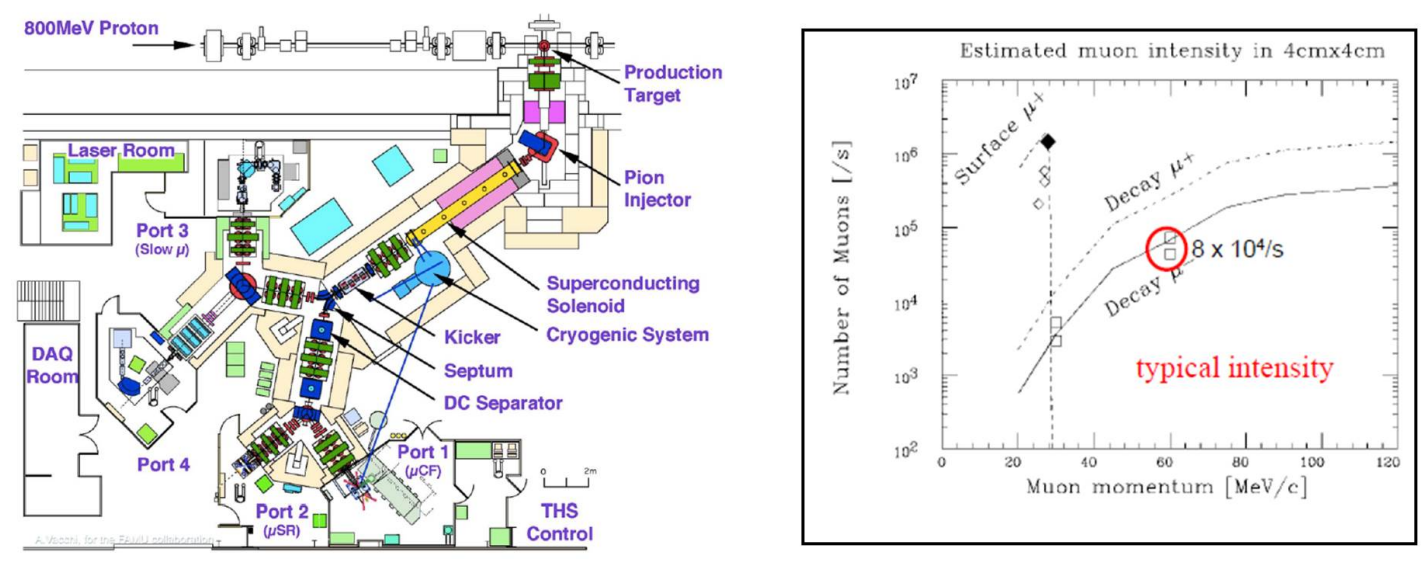

Figure 2. Left panel: schematic layout of the RIKEN-RAL muon facility at RAL, with its four experimental ports. The FAMU experiment presently uses port 4 and will move soon to port 1 for its final run. Right panel: estimated intensity in a $4 \times 4 \mathrm{~cm}^{2}$ area for surface and decay muon beams at RIKEN-RAL.

\section{Construction of the R484 and $\mathbf{R 5 8 2}$ beam hodoscopes}

At the entrance of the FAMU target, the beam profile is nearly flat (see later) and thus we may expect $\sim 20 \mu / \mathrm{mm}$ in the $70 \mathrm{~ns}$ long spill (along transverse $\mathrm{x} / \mathrm{y}$ directions). This fact puts severe constraints on the beam hodoscope construction and prevents a particle by particle identification.

\subsection{The $\mathbf{R 4 8 4}$ experiment beam hodoscope}

The R484 beam hodoscope (see figures 3 and 4 for details) consists of 32+32 scintillating fibers with square cross-section ${ }^{1}$ arranged along $\mathrm{X} / \mathrm{Y}$ axis (orthogonal axis perpendicular to the beamline). The use of square fibers makes the detector response independent from the position of the muon trajectory inside a fiber and minimizes the amount of dead spaces. The fibers have been cut to

\footnotetext{
${ }^{1}$ Bicron BCF12 single clad, side $3 \mathrm{~mm}$, peak emission $\sim 435 \mathrm{~nm}$, trapping efficiency $\sim 7 \%$, attenuation length 270 $\mathrm{cm}$, decay time $\sim 3.2 \mathrm{~ns}$, light yield $\sim 8000$ photons $/ \mathrm{MeV}$
} 
a length of $\sim 102.5 \mathrm{~mm}$ with a Fiberfin 4 machine at CERN, that provides directly polished ends ready to use, suited for a detector with a $10 \times 10 \mathrm{~cm}^{2}$ fiducial area. The fiber thickness of $3+3$ $\mathrm{mm}$ corresponds approximately to $\sim 7 \%$ of the range of a $60 \mathrm{MeV} / \mathrm{c}$ muon. A muon passing through a fiber produces scintillation light that is detected at one end of the fiber by a $3 \times 3 \mathrm{~cm}^{2}$ Silicon photomultiplier $(\mathrm{SiPM})^{2}$. For space problems, as the SiPM package footprint is bigger than the fiber size, fibers are read from one edge only, alternating left/right (fibers along X-axis) and up/down (fibers along Y-axis) sides. Fibers were wrapped with an Al-film, $10 \mu \mathrm{m}$ thick, to avoid channel to channel light cross-talk. Single RG174 cables, with MCX connectors on one side and LEMO00 connectors on the other side, convey both the SiPM's bias (on the external braid shield) and signal ouput (on the cable inner connector). In this way, each SiPM may be powered individually. Each SiPM is mounted on a custom printed board (in groups of 16), as shown in figure 4. SiPMs were hand-soldered on one side of the PCB, facing the scintillating fibers, while MCX connectors were mounted on the other side of the PCB. The detector is housed in a 3D printed ABS case ${ }^{3}$. The choice of Advansid RGB SiPMs ${ }^{4}$ as photodetectors for the R484 beam hodoscope
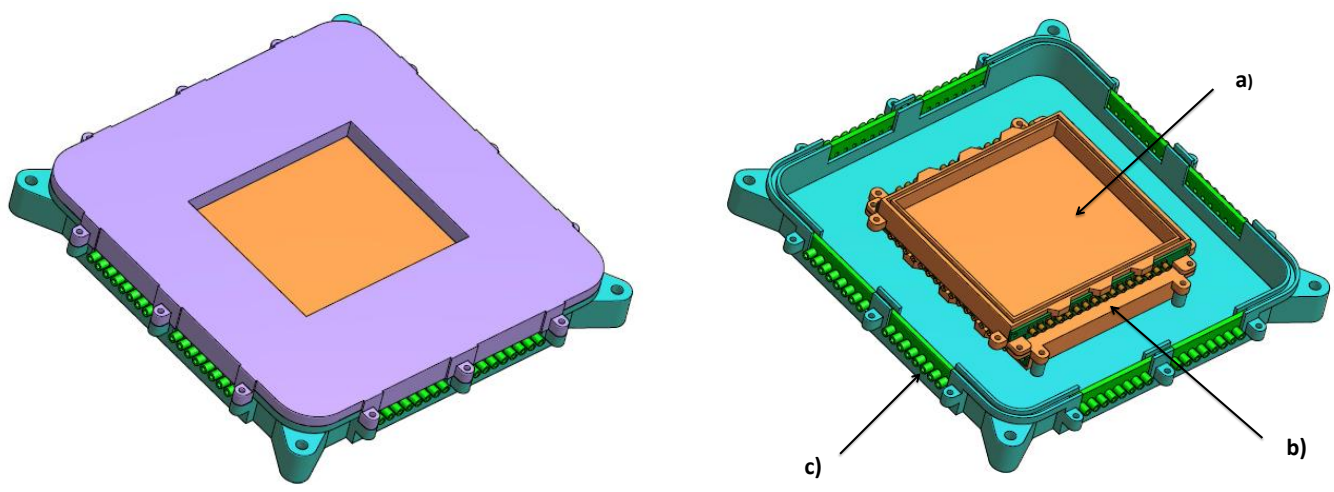

Figure 3. Mechanics layout of the R484 $3 \mathrm{~mm}$ pitch beam hodoscope. Left panel: general structure of the light-tight assembly. Right panel: details of one of the two cover boxes. a) holder for the scintillating fibers; b) custom PCB with SiPMs on one side (towards the fiber face) and MCX connectors, to convey signal and power for each individual channel, on the other side; c) cable feed-through on the lateral side of the structure.

was dictated by their short pulse duration, their high photon efficiency, well matched to the BC12 fiber peak emission (PDE $\sim 22 \%$ at $\sim 440 \mathrm{~nm}$, with $4 \mathrm{~V}$ overvoltage), their low operating voltage $\left(V_{b r k} \sim 29 \mathrm{~V}\right)$, their small breakdown voltage dependence from temperature $(\sim 27 \mathrm{mV} / \mathrm{C})$ and their low dark noise, see figure 5 for details. All $3 \times 3 \mathrm{~mm}^{2}$ SiPMs were individually charaterized to determine their breakdown voltage by measuring their current-voltage charateristic, as shown in the right panel of figure 5 .

The detector front-end was based on refurbished electronic boards from the INFN TPS project [5], that provide power to single SiPMs (up to $40 \mathrm{~V}$ ). This feature allowed to use only Advansid or SENSL SiPMs, excluding Hamamatsu or KETEC ones, that needed higher voltages. The TPS

${ }^{2}$ Advansid RGB type, with $40 \mu \mathrm{m}$ cells

${ }^{3}$ printed on a Stratasys Elite Dimension printer, with $0.18 \mathrm{~mm}$ resolution

${ }^{4} \mathrm{ASD}-\mathrm{SiPM} 3 \mathrm{~S}-\mathrm{P} 40$, in a production batch, with a $\sim 600 \mathrm{~K} \Omega$ quenching resistor 


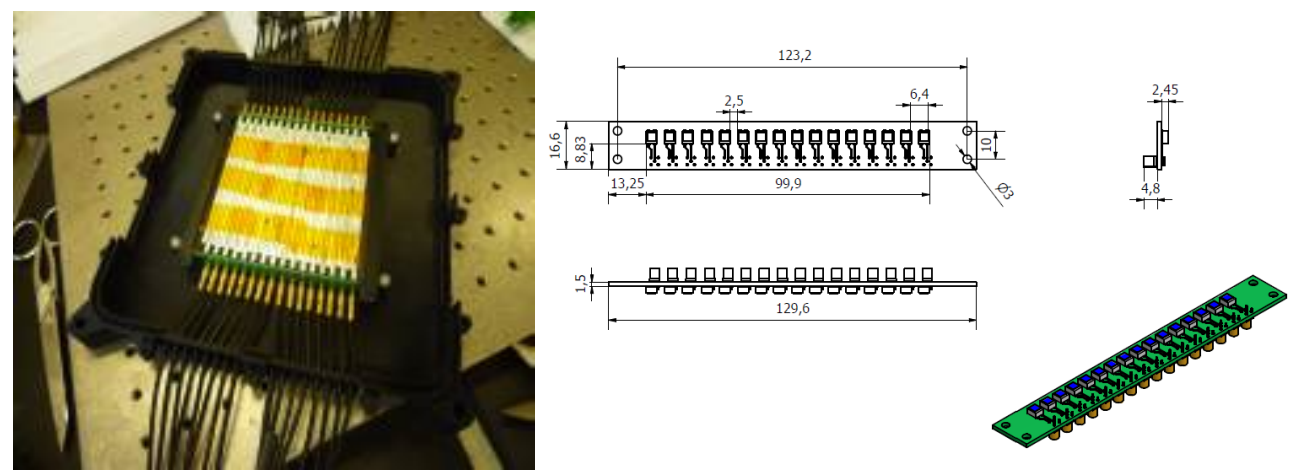

Figure 4. Left panel: hodoscope assembly: one layer of fiber is visible, inside the fiber holder. Each fiber is facing a SiPM mounted on one side of a custom PCB. All is already housed inside one of the two ABS cover boxes. RG174 cables, with MCX connectors on one side, are visible and extend beyond the cover box volume, through feedthrougs. Right panel: schematic layout of the custom PCB holding on the top side the 16 SiPMs, facing fiber edges and on the bottom side MCX connectors.
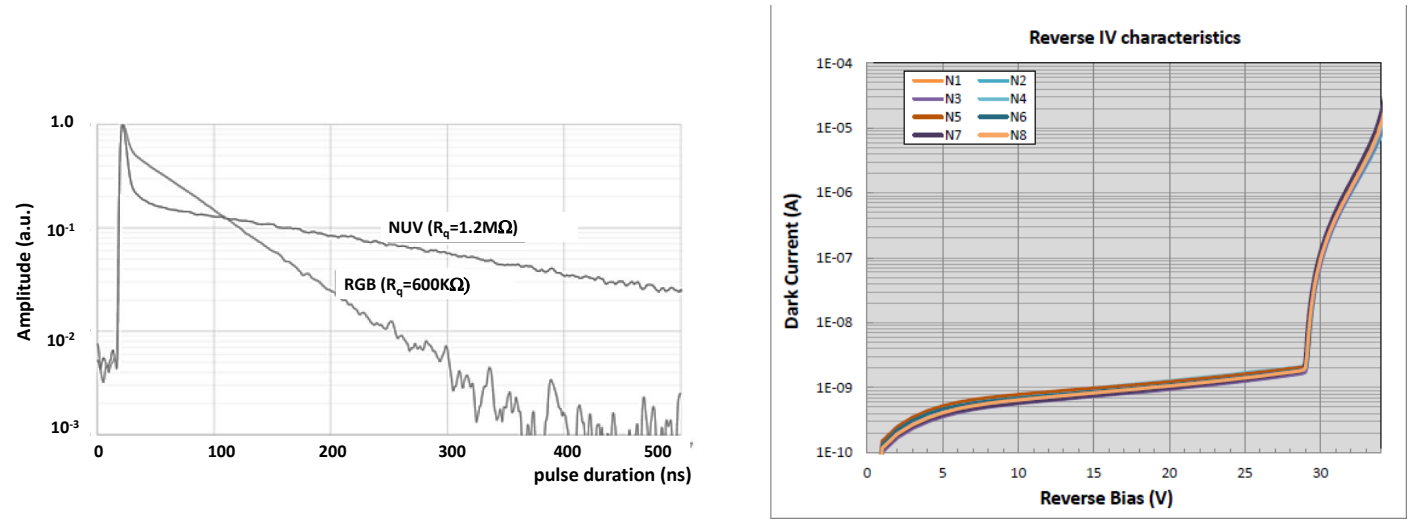

Figure 5. Left panel: pulse duration for RGB and NUV types of Advansid $3 \times 3 \mathrm{~mm}^{2} \mathrm{SiPMs}$. The shorter pulse duration of the RGB type ( $200 \mathrm{~ns}$ ) has to be noticed [courtesy of Advansid srl]. Right panel: reverse current-voltage characteristic for a sample of Advansid RGB SiPMs. The breakdown voltage is estimated around 29.1 V. Measurements were done at $T_{A}=25^{\circ} \mathrm{C}$.

electronic system is based on a VME-like crate, providing power to custom boards and a GPIB module interface. Each TPS electronics board, with 8 individual channels, provided individual channel voltage fine regulation, signal amplification and shaping, signal discrimination and trigger capability, by using the OR of the eight channels of a board. Output signals are also fed into a CAEN V792 QDC for measurement of the charge integrated signal.

The beam hodoscope has been first tested with cosmics at INFN Milano Bicocca, where it has been built, and at the Beam Test Facility (BTF) of the INFN LNF laboratory [6], before the R484 data-taking in mid 2014 at RAL. A custom data-acquisition system was based at first on the Bit3 VME-PCI interface and afterwards on the CAEN V2718 VME-PCI interface. Test beam results at BTF with impinging electrons ${ }^{5}$, already reported in reference [7], show a signal to noise ratio

$5490 \mathrm{MeV}$ kinetic energy, nominal beam profile $9 \times 7 \mathrm{~mm}^{2}$, beam multiplicity 5 particles/spill 
better than 10 and a single to double MIP separation at $\sim 1.5 \sigma$. With $60 \mathrm{MeV}$ muons, we expect $\sim 300$ photons/muon arriving at the $3 \times 3 \mathrm{~mm}^{2}$ window of a SiPM, giving a collected charge of $\sim 7-14 \mathrm{pC}$ at the QADC input, for a nominal SiPM's gain of about $0.8-1.5 \times 10^{6}$. In order to match the V792 QADC range (0-400 pC), with the foreseen muon rate at RIKEN-RAL and avoid saturation, 10x attenuators had to be used before the QDC input.

\subsection{The $\mathbf{R 5 8 2}$ experiment beam hodoscope}

In the following R582 experiment at RIKEN-RAL, a $1 \mathrm{~mm}$ pitch beam hodoscope, to be put in front of the new cryogenic target, was developed. The main requirements were to reduce to a minimum

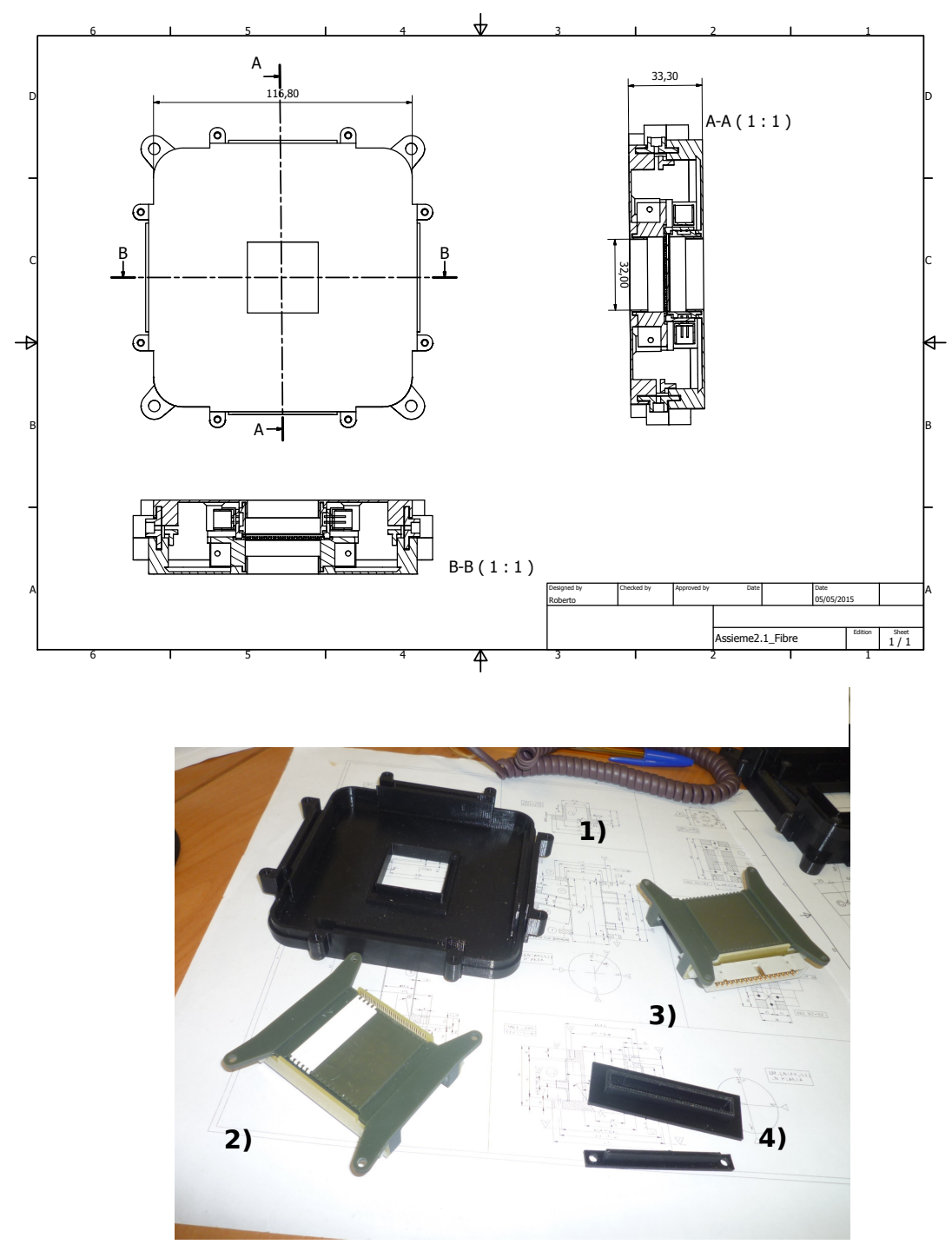

Figure 6. Top panel: CAD design of the $1 \mathrm{~mm}$ pitch hodoscope, with front and side views. Bottom panel: main mechanical components of the detector. Item 1) is one of the two light-tight beam hodoscope cover boxes; item 2),3) are $\mathrm{X} / \mathrm{Y}$ plane fiber holders: some fibers are mounted in 2); 4) is the cable pass-through.

the material in front of the target thin Be window and to try to simplify the layout of the front-end 
electronics based on one side on VME-like custom TPS boards, with a GPIB to USB control module and on the other side on VME-compliant QDAC and TDCs. To solve the first issue, $32+321 \mathrm{~mm}^{2}$ square scintillating Bicron BCF12 fibers ${ }^{6}$ were used. They were arranged in two orthogonal planes along $\mathrm{X} / \mathrm{Y}$ coordinates, giving a detector fiducial area of $32 \times 32 \mathrm{~mm}^{2}$. As before, fibers were cut on a Fiberfin-4 machine at Cern, giving ready-to-use fibers with polished ends. The mechanics layout and the main mechanical components are shown in figure 6 . The reduced size of mechanics details of some components gave severe problems for the 3D-printing: issues were solved using an ENVISIONTEC Perfactory 3D-printer at LNGS with a precision better than $0.025 \mathrm{~mm} .1 \times 1 \mathrm{~mm}^{2}$
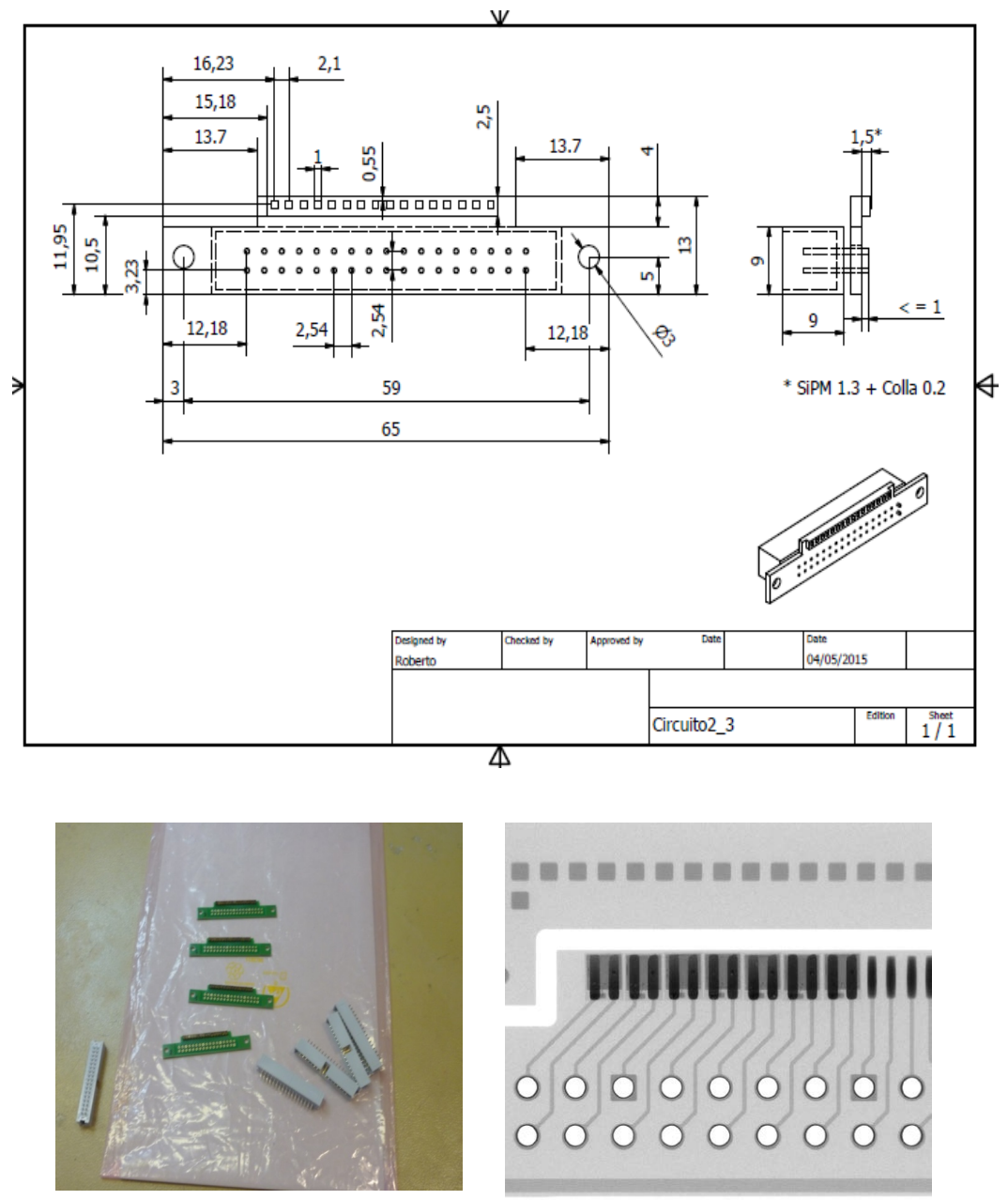

Figure 7. Top panel: schematic layout of the PCB holding on one side the 16 SiPMs facing the fibers and on the other side the 34-way flat-cable connector. This connector has a size comparable to the one of the PCB where it is mounted. Right bottom panel: X-rays image to cross-check the SiPMs mounting on the PCB. Left bottom panel: picture of some PCBs and the 34-way connectors.

${ }^{6}$ coated with white Extra Mural Absorber (EMA), to avoid light cross-talk 
square SiPMs from Advansid ${ }^{7}$ were used to detect scintillation light emitted by crossing muons in the detector's fibers. As the SiPM's footprint is slightly bigger than the fiber cross-section, fibers had to be read alternating left/right and up/down sides, as for the R484 hodoscope. Each SiPM is mounted on a custom PCB (in groups of 16) as shown in figure 7. On the side facing the fibers the SiPMs have been soldered ${ }^{8}$, while on the opposite side a 34-way flat-cable connector was mounted, to convey bias and take out signals from the SiPMs. Some steps of the mounting of the detector are shown in figure 8, starting from the holder of one plane of fibers to the installation at RIKEN-RAL. Advansid RGB $1 \times 1 \mathrm{~mm}^{2} \mathrm{SiPMs}$ were chosen as photodetectors for the same reasons that dictated
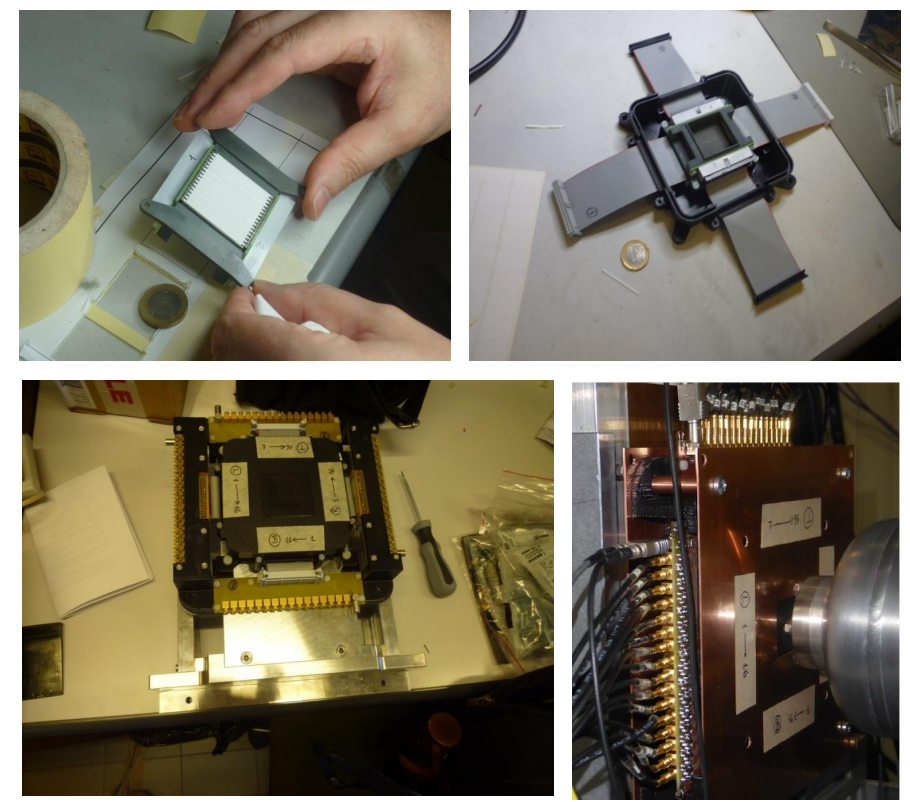

Figure 8. Left-top panel: holder of one plane of fibers, with white EMA coated fibers inserted. Right-top panel: mounted detector, without the cover box. Flat cables extend outside. Left-bottom panel: mounted detector, with an interface board visible on the bottom. Right-bottom panel: complete detector, with $1.5 \mathrm{~mm}$ copper planes for electrical shielding installed at RIKEN-RAL. The cryogenic target nose is visible on the right.

the choice for the $3 \times 3 \mathrm{~mm}^{2} \mathrm{SiPM}$ of the R484 beam hodoscope. All available SiPMs (115 in all) were tested individually to determine their breakdown voltages by measuring their current-voltage characteristic, see the left panel of figure 9 for an example. The right panel of figure 9 shows the distribution of their breakdown voltages: on average $28.94 \pm 0.08 \mathrm{~V}$. It was thus possible, with a suitable selection of the SiPMs to be used, to employ a common voltage for the biasing of the SiPMs of a detector plane. We checked that the 34-way flat cable, that had not a $50 \Omega$ impedance, gave no appreciable distortions to the signal, thanks to its short length. Figure 10 shows one of the four interface boards, with the flat cable socket on one side and angled MCX connectors for signal

\footnotetext{
$740 \mu \mathrm{m}$ cells, RGB type

${ }^{8}$ The mounting was realized at Mevinco srl, Soiano del Lago, Brescia and cross-checked with X-ray imaging, see the right panel of figure 7 for details
} 

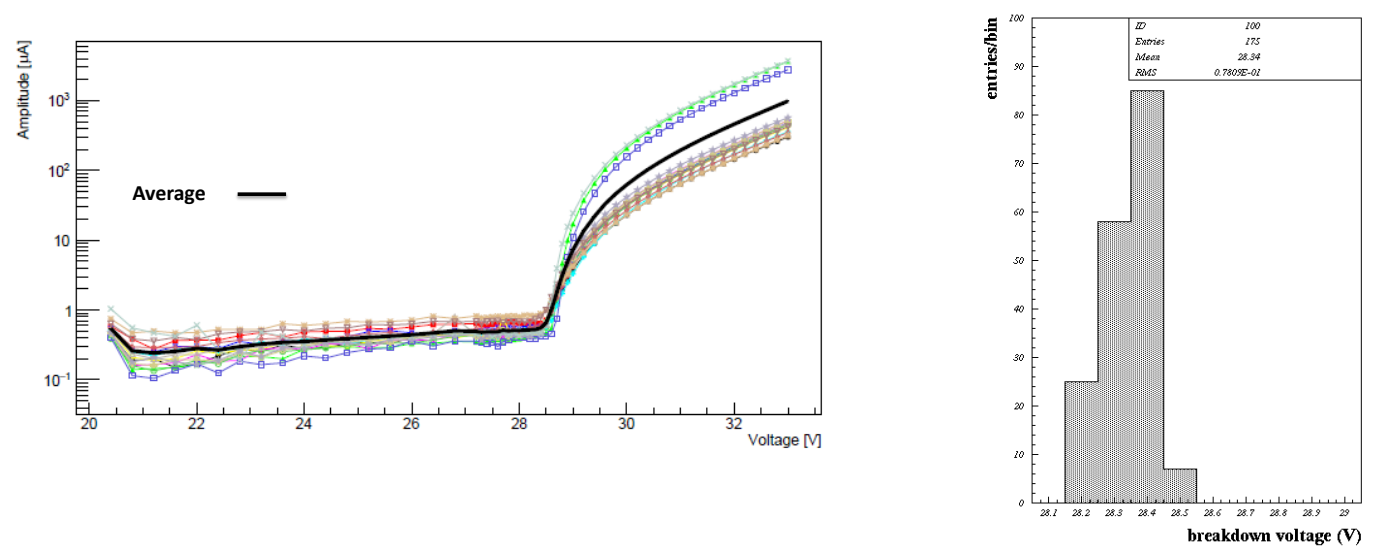

Figure 9. Left panel: reverse I-V characteristic for the sample of $1 \times 1 \mathrm{~mm}^{2} \mathrm{SiPMs}$ mounted on one side of the hodoscope. Right panel: distribution of $V_{b r k}$ for the sample of $1 \times 1 \mathrm{~mm}^{2} \mathrm{SiPMs}$.

cables and one LEMO 00 connector for the power line on the other side. Signals are then fed into a

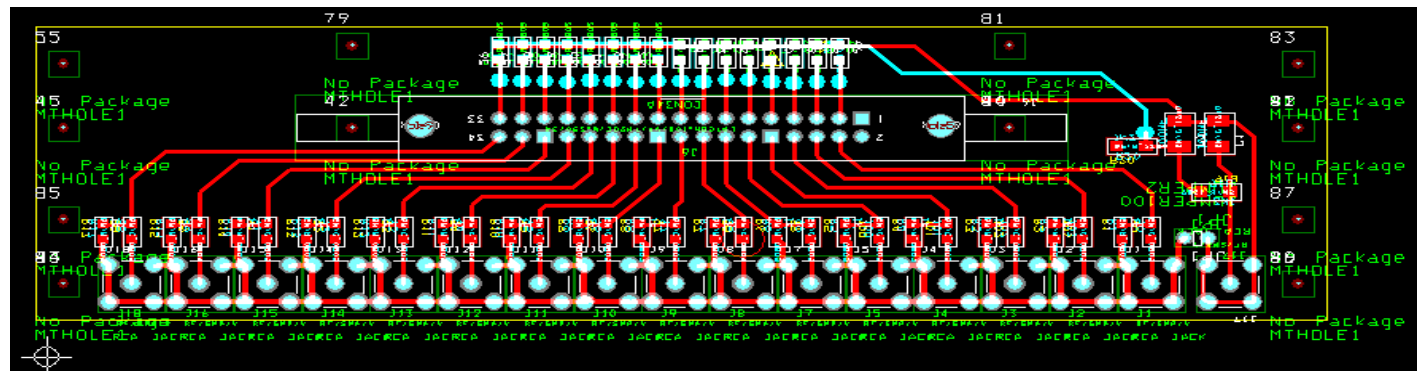

Figure 10. Electronics rendering (GERBER) of the interface board for the beam hodoscope of R584. The 34-way flat cable connector is placed on top.

fast FADC ${ }^{9}$ operated at a reduced sampling rate of $1 \mathrm{Gs} / \mathrm{s}$ to increase the digitizing buffer time.

Foreseen developments include the addition of onboard thermistors to ensure long-term stability via correction of SiPMs gain thermal drift and the possibility to use also timing informations from the detector and the monitor of the beam intensity. The left panel of figure 12 shows the FADC waveform for a typical channel, where the two pulses beam structure is clearly visible. Signals were then processed via a custom DAQ sysyem based on a CAEN V2718 VME-PCI interface.

\section{Preliminary performances at RIKEN-RAL.}

The two beam monitors have been used at RIKEN-RAL to optimize the beam steering inside the target. For the R484 experiment, the integrated charge provided by the CAEN V792 QDC was directly used, while for the R582 experiment the signal waveform for each channel was integrated, after subtracting the baseline, providing the same type of infos. Results for a typical run in R484

${ }^{9} \mathrm{CAEN}$ V1742 FAC with $5 \mathrm{GS} / \mathrm{s}, 12$ bit, 1 Vpp input dynamic range, in VME standard 

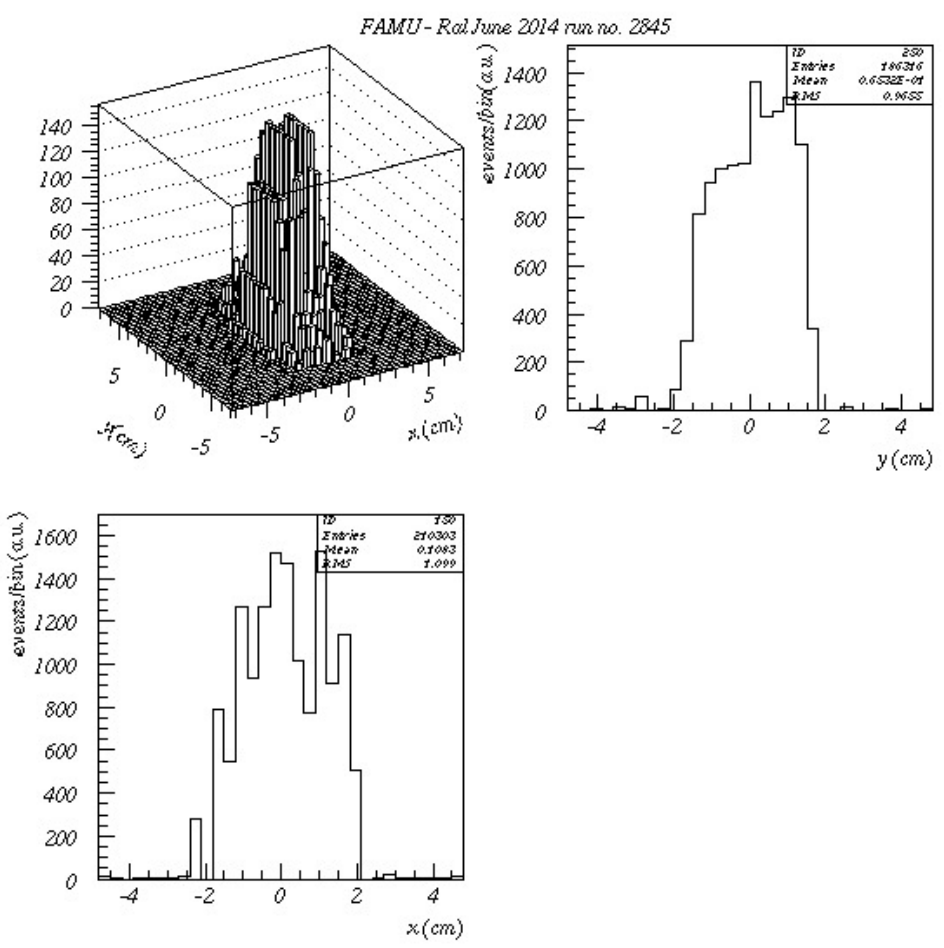

Figure 11. $\mathrm{X} / \mathrm{Y}$ beam profile for a nominal optics run in $\mathrm{R} 484$ at $61 \mathrm{MeV} / \mathrm{c}$.

are shown in figure 11, while results with standard optics for the R582 run are shown in figure 12. In both cases the beam size is defined by the collimator aperture, with a measured RMS smaller than $10 \mathrm{~mm}$.
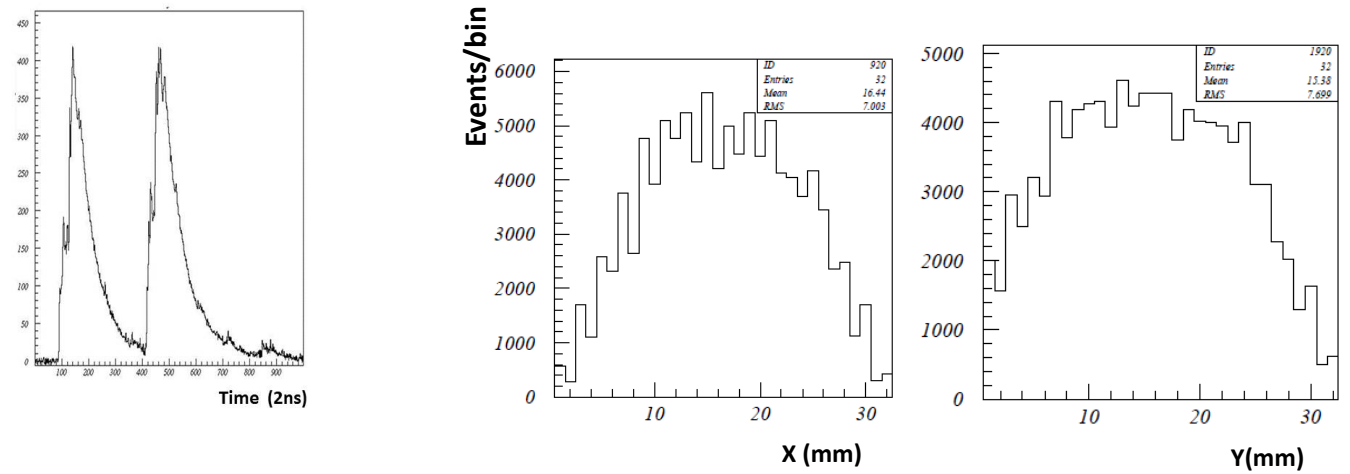

Figure 12. Left panel: FADC waveform for a typical beam hodoscope channel. Right panel: $X / Y$ beam profile at RIKEN-RAL for a $60 \mathrm{MeV} / \mathrm{c}$ run. 


\section{Conclusions}

Two scintillating fibers beam hodoscopes with $1 \mathrm{~mm}$ and $3 \mathrm{~mm}$ pitch and X/Y readout by SiPM have been developed and used for the measurement of 50-60 MeV/c beam profiles at RIKEN-RAL muon beams. Performances in agreement with expectations are reported and foreseen developments include the possibility to monitor the beam intensity and use timing informations.

\section{Acknowledgements}

We would like to thank S. Banfi, M. Geigher (INFN Milano Bicocca), O.Barnaba,R.Nardó (INFN Pavia), A. Iaciofano (INFN Roma Tre) for help in mechanics and electronics. We acknowledge the help of T.Schneider (CERN) for fiber cutting at CERN, D. Orlandi (LNGS) for 3D-printing of some items of the detector at LNGS, N. Serra (Advansid) for SiPMs best handling and of P.Branchini and D.Tagnani (INFN Roma Tre) for the optimal use of TPS electronics . We would like to thank also the staff of ISIS and the RIKEN-RAL facility for the generous help during data-taking and in particular of K. Ishida for advice in the optimal use of our detectors in beam.

\section{References}

[1] A. Vacchi et al., Measuring the size of the proton SPIE Newsroom (2012) DOI:10.1117/2.1201207.004274;

A. Adamczack et al., Steps towards the hyperfine splitting measurement of the muonic hydrogen ground state: pulsed muon beam and detection system characterization, JINST 11/05 (2016) P05007; A. Adamczak et al., Theoretical and computational study of the energy dependence of the muon transfer rate from hydrogen to higher Z-gases, Phys. Lett. A379 (2015) 151;

M. Bonesini, The proton radius puzzle, contribution to ICFNP 2016, to be published on EPJ web of Physics

[2] A. Adamczack et al., Hyperfine spectroscopy of muonic hydrogen and the PSI Lamb Shift experiment, Nucl. Instr. Meth. B281 (2012) 72;

D. Bakalov et al., Experimental method to measure the hyperfine splitting of muonic hydrogen $\left(\mu^{-} p\right)_{1 S}$, Phys. Lett. A172 (1993) 277;

[3] A.C. Zemach Proton Structure and the Hyperfine Shift in Hydrogen, Phys. ReV. 104 (1956) 1771.

[4] R.Pohl et al., The size of the proton Nature 466 (2010) 213;

A. Antognini et al. Proton Structure from the Measurement of $2 S-2 P$ Transition Frequencies of Muonic Hydrogen, Science 339 (2013) 417

[5] P. Branchini et al. An FPGA based general purpose DAQ module for the KLOE-2 experiment, IEEE Trans. Nucl. Sci. 58 (2011) 1544;

R. Pleskac et al. The FIRST experiment at GSI, Nucl. Instr. Meth. A678 (2012) 130.

[6] G. Mazzitelli et al., Commissioning of the DAФNE beam test facility, Nucl. Instr. Meth. A515 (2003) 524.

[7] R. Carbone et al., The fiber-SiPM beam monitor of the R484 experiment of the RIKEN-RAL muon facility, JINST 10 (2015) C03007 